\title{
Hypertensive Microbleed as a Transient Ischemic Attack Mimic
}

\author{
Claus Z. Simonsen ${ }^{\mathrm{a}}$ Edith Nielsen ${ }^{\mathrm{b}}$ \\ Departments of ${ }^{a}$ Neurology and ${ }^{b}$ Neuroradiology, Aarhus University Hospital, Aarhus, \\ Denmark
}

\section{Key Words}

Stroke $\cdot$ Transient ischemic attack mimic $\cdot$ Microbleed $\cdot$ Hypertension

\begin{abstract}
Cerebral microbleeds have acquired increased attention as a silent marker of small vessel disease that carries an increased risk of hemorrhage. The etiology is believed to be either hypertension or amyloid deposition. Here, we present a case with a patient whose transient focal symptom most likely was due to the occurrence of an acute microbleed, indicating that not all microbleeds are silent and that the cause of a transient ischemic attack is not always ischemic.
\end{abstract}

While driving her car, a 46-year-old woman with long-standing idiopathic hypertension developed a sudden tingling sensation in the right side of her face and arm, heaviness of the right arm, double vision and nausea. Notably, her hypertension was diagnosed at the age of 16 years, and her blood pressure had been difficult to control. At the time of presentation, she was taking three anti-hypertensive drugs. She had never experienced neurological symptoms before.

She drove to an emergency department where mild weakness of the right arm was found, and she was immediately referred to the stroke department. An MRI scan revealed no acute ischemia on the diffusion-weighted scan, but on T2*-weighted imaging, a dark focus of increased susceptibility ('microbleed') was visible in the left pons, in the area of her described symptoms (fig. 1). The symptoms fit well with the location because of the proximity to the corticospinal tract (lesion of which causes contralateral weakness) and the medial longitudinal fasciculus (lesion of which causes double vision due to internuclear ophthalmoplegia.) Dizziness is a rather unspecific symptom but often seen with lesions in

Claus Z. Simonsen, MD, PhD

Department of Neurology

Aarhus University Hospital, Nørrebrogade 44

DK-8000 Aarhus C (Denmark)

E-Mail clasim@rm.dk 


\section{Case Reports in Neurology}

\begin{tabular}{l|l}
\hline Case Rep Neurol 2013;5:31-33 & \\
\hline DOI: $\underline{10.1159 / 000348400}$ & $\begin{array}{l}\text { ○ 2013 S. Karger AG, Basel } \\
\text { www.karger.com/crn }\end{array}$ \\
\hline
\end{tabular}

Simonsen et al.: Hypertensive Microbleed as a Transient Ischemic Attack Mimic

the vertebrobasilar circulation. On the B-zero diffusion series, the microbleed revealed a rim of T2 hyperintensity consistent with edema and an acute timing of the lesion (fig. 2).

On the following day, the patient complained of subjective dizziness, but the neurological exam was normal. She was referred to the nephrology service for better antihypertensive control and was subsequently discharged.

Transient ischemic attack (TIA) is defined as a focal neurological deficit that normalizes within $24 \mathrm{~h}$ and is attributable to ischemia. Microbleeds have been associated with transient deficits, but only in patients with cerebral amyloid angiopathy $[1,2]$. This is, to our knowledge, the first report of a presumed hypertensive microbleed presenting with TIA-like symptoms. The case emphasizes the usefulness of MRI in the workup of TIA patients.

\section{References}

1 Watanabe A, Kobashi T: Lateral gaze disturbance due to cerebral microbleed in the medial lemniscus in the mid-pons region: a case report. Neuroradiology 2005;47:908-911.

2 Greenberg SM, Vonsattel JP, Stakes JW, Gruber M, Finkelstein SP: The clinical spectrum of cerebral amyloid angiopathy: presentations without lobar hemorrhage. Neurology 1993;43:2073-2079.

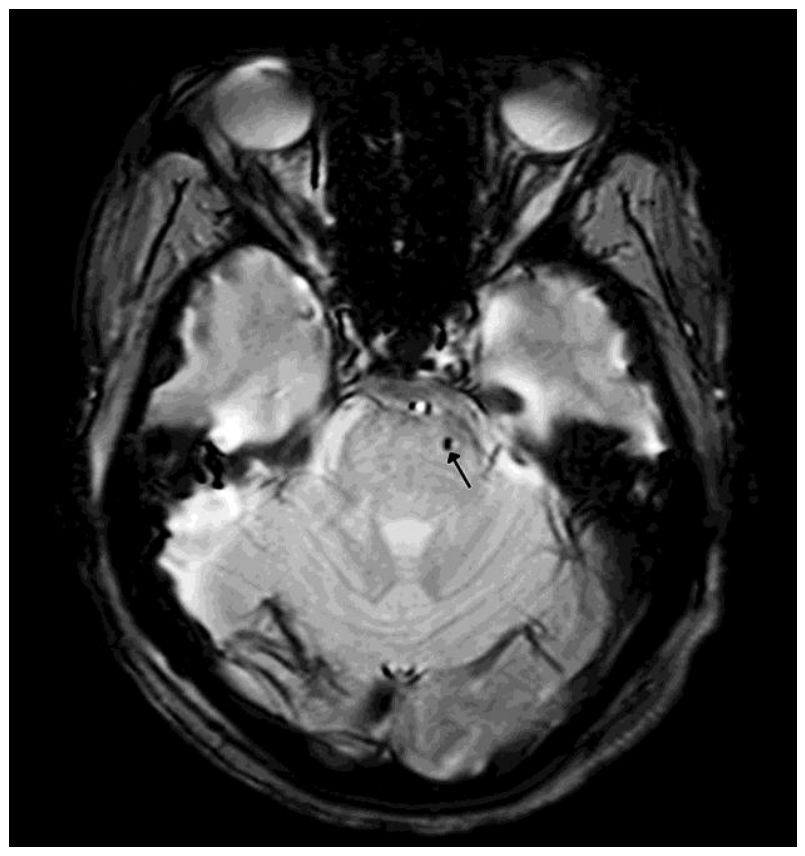

Fig. 1. T2*-weighted image. The arrow indicates the presence of a microbleed in the left pons. 


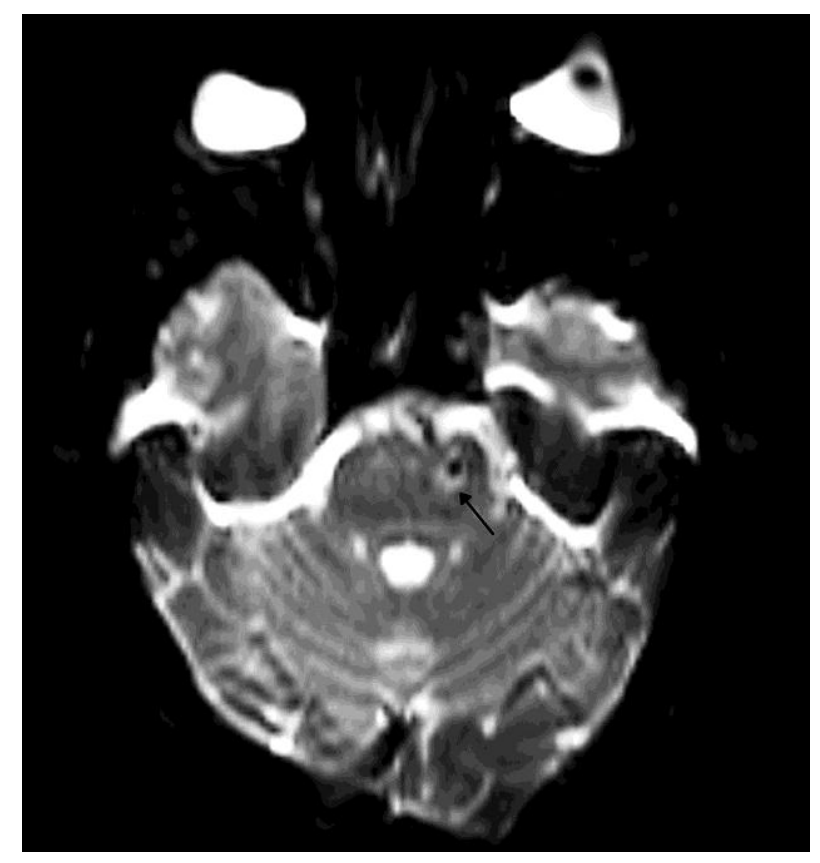

Fig. 2. Unweighted image $(b=0)$ in the diffusion-weighted series. In effect it is a T2-weighted image. The arrow points to the bright rim (edema) around the lesion, indicating that the lesion is acute. 